\title{
"What is The SI?” A Proposal for an Educational Adjunct to the Redefinition of the International System of Units
}

\author{
Neil M. Zimmerman and David B. Newell \\ National Institute of Standards and Technology, \\ Gaithersburg, MD 20899 \\ neilz@nist.gov, +1 3019755887 \\ david.newell@nist.gov
}

\begin{abstract}
We discuss how the impending redefinition of the SI system of units might affect the ability of students to understand the link between the units and the new system. The redefinition will no longer define a set of base units, but rather a set of constants of nature, such as the elementary charge, $e$. We point out that this list of constants need not be the only way to introduce students to the subject, either in class or in textbooks. We suggest an alternative way to introduce high school and undergraduate students to the redefined SI, by suggesting a list of experiments for some units; this list would be completely compatible with the redefined SI, and would have all of the same scientific and technological advantages. We demonstrate by questionnaire results that this alternative is more appealing to students. We hope to spur a discussion amongst teachers regarding this important topic for high school and undergraduate physics courses.
\end{abstract}

Key words: International System of units; redefinition; student education.

Accepted: November 10, 2018

Published: December 14, 2018

https://doi.org/10.6028/jres.123.024

\section{Introduction and Motivation}

The International System of Units (SI or Système International d'Unités) is scheduled to be fundamentally changed on 20 May 2019 [1-3]. One aspect of the fundamental change is that the SI will no longer be defined in terms of experiments that lead to the units, but rather with respect to a set of defining constants of nature. We are concerned about the effect of this change on the education of beginning (senior high school and early college) science, technology, engineering, and mathematics (STEM) students, because we believe it is easier for students early in their career to grasp the concept of units as being based on experiments.

Before we jump into a detailed discussion of the redefinition of the SI, we will give a general introduction into the need for any system of units, and how the present SI evolved. It could be argued that the need for physical measurements has existed at least since we had to judge where to place one foot in front of the other in the controlled fall known as walking. Indeed, we perform measurements all the time subconsciously as we estimate or judge physical quantities surrounding us, such as length, size, weight, or speed. With the development and advancement of the first ancient agricultural societies came the need to standardize physical measurements to facilitate trade and commerce. It is interesting to note that at the foundation of these early measurement systems was a "universal measure" that was available to everyone, 
anytime, anywhere, such as a seed or a grain, from which measurement units related to length, area, volume and mass were constructed [4].

The existing SI is also based on perceived universal measures dating back to the formation of the metric system during the French revolution. Back then, the length of Earth's meridian was chosen as a constant of nature or a universal measure on which an everyday practical unit of length, the meter, was based [5]. Derived from the meter was the practical unit of mass, the kilogram, based upon a certain volume $(0.001$ $\mathrm{m}^{3}$ ) of distilled water close to the freezing point. Together the meter, kilogram, and second (being based on astronomical observations) provided a practical system of units not only for measuring everyday physical quantities used in trade and commerce, but also for measuring newly discovered phenomena such as electricity and magnetism.

Today, the present SI defines explicitly seven base units, of which the most familiar are the meter, kilogram, and second. Not only are they used for everyday practical measurements, but, together with simple dynamics and Newtonian physics, they are used as educational tools for introducing the system of units. In addition, with the exception of the kilogram [6], the definitions of the other six present-day SI base units have the effect of implicitly defining the values of six true invariants of nature (which thus have zero uncertainty), such as the hyperfine frequency of cesium atoms $\Delta v_{\mathrm{Cs}}$ and the speed of light $c$; in contrast, other fundamental constants such as Planck's constant $h$ are not defined, and thus are measured with nonzero uncertainty.

There is an international agreement to redefine the SI so that it defines explicitly seven defining constants [1-3]. This will have the effect of implicitly defining a large number of possible experiments which could be used to realize the various units. The basic motivations for the change can roughly be distilled as: (1) replacing the only remaining artifact standard (i.e., a standard based on a particular piece of metal located in Paris, France), the International Prototype of the Kilogram (the IPK), with a definition of mass based on fundamental laws of physics; (2) using the excellent reliability and reproducibility of the quantum electrical standards; (3) defining the SI explicitly in terms of fundamental constants (the "defining constants") rather than base units.

We note several excellent resources for detailed discussions of this variety of motivations: For discussions of mass including the present system and the possible experiments in the new SI (the "electronic kilogram" and a nearly-perfect Si sphere), please see Ref. [6]. For a discussion of the system of electrical units including the "quantum electrical standards" (Josephson voltage and quantum Hall resistance standards), please see Ref. [7]. A discussion of the physics of the defining constants, and the ways in which the best values are periodically achieved, is given in Ref. [8]. Finally, technical discussions of best practices for realizing many of the units are discussed in the "mises en pratique" [9].

While the motivations and the practicality of the redefinition seem to us to be quite compelling for practitioners in metrology (the science of measurement), we believe that presenting the new definition of the SI strictly in terms of exact values of defining constants [motivation (3)] may not be the best way to introduce the topic of the new SI to students. There are several ways that we can make this point: (1) The "International System of Units" has the word units in it, and many students may find confusing a System of Units that has no units named in it [3]; (2) As pointed out by one of us [2], it may be quite challenging for teachers of senior high school students or beginning college students to explain how the unit of mass flows from the value of Planck's constant $h$, which is typically not introduced until much later in a student's career; (3) Similarly, many physics textbooks have a discussion of or an appendix on units, and explaining concisely how (for example) time depends on a particular atomic frequency (see definition A in Fig. 1) may again be quite challenging.

There are many advantages to the likely redefinition of the SI, including (1) replacing the IPK; (2) having a system of units that depends only on fundamental laws and invariants of nature; (3) making it 
possible for all units (not just the seven base units) to be expressed in terms of seven defining constants which are defined to have zero uncertainty [for example, in temperature the SI unit kelvin would be $1 \mathrm{~K}=$ $2.266665\left(h \Delta v_{\mathrm{Cs}} / k\right)$, where $k$ is the Boltzmann constant]; (4) eliminating the "1990 conventional electrical units", which refers to the fact that for the past 25 years, electrical metrologists have used the quantum electrical standards (Josephson voltage standard and quantum Hall resistance standard) [7] to disseminate electrical standards, which means that they are not reporting their results in SI units [10] but rather in the so-called "1990 units"; (5) by virtue of the seven defined constants, allowing scaling of definitions of units over many orders of magnitude.

In the proposal contained in this paper, we suggest that students first be introduced to the SI in terms of units rather than defined constants. We wish to emphasize that this proposed educational adjunct to the SI is completely compatible with the scheduled 2019 redefinition; in particular, all of the advantages listed above for the scheduled redefinition are also reflected in our proposed introduction based on units. We also wish to emphasize that our proposal is not for a definition of the SI, but rather for the use of teachers in introducing the redefined SI to students, either in classes or in textbooks.

\section{Three Possible Introductions to the SI}

We refer the reader to Figures 1, 2, 3; these figures contain three possible versions of the text that could be used to introduce students to the subject of units. Briefly, proposal A is quoted from the draft ninth edition of the SI brochure [1] [from here on we refer to this as the " $9^{\text {th }}$ SI brochure"], and sets out the definitions and values of the seven defined constants; proposal B is adapted from a different Section of the

$9^{\text {th }}$ SI brochure, and lists the seven traditional base units as defined in terms of the seven constants; proposal $\mathrm{C}$ is an alternative proposal, and contains a list of what we believe to be the more commonly-used units, defined not in terms of constants but in terms of brief descriptions of experiments that could be used to realize those units. As discussed at the end of the Introduction and Motivation, we note that these three proposals are interchangeable in terms of both the effect on the system of units, and on satisfying all the advantages listed above. In all three proposals, we added the same introductory paragraph for the purposes of the questionnaire; this paragraph does not appear in the $9^{\text {th }}$ SI brochure.

We now discuss how we derived these three proposals: proposal A is quoted from the $9^{\text {th }}$ SI brochure [1], and we suspect is what most metrologists who have been involved in the likely redefinition would answer when asked: "What is the SI?". Proposal C is an attempt to address the motivation that we have discussed, in terms of introducing students to the units based on simple descriptions of the types of experiments that one might use to realize those units. We discuss how we arrived at this proposal in more detail directly below.

As with proposal A, proposal B is taken from a different section of (a previous version of) the $9^{\text {th }}$ SI brochure and was thus devised by the metrologists who are advocating the likely redefinition; in the new version dated 5 February 2018, this section is quite similar, but now has references to other units [1]. It has a list of units, but the definitions are based on invariants of nature and not on experimental realizations; it thus reflects some of the attributes of the two other proposals.

Turning in more detail to proposal $\mathrm{C}$, we will note that we took this proposal through several versions. In an earlier, longer version of this proposal, we had much more detail. Although the longer proposal $\mathrm{C}$ was favored by students in a questionnaire comparing their understanding of the motivation underlying each proposal (see Appendix), a significant number of them also commented that the original proposal $\mathrm{C}$ was too long and complicated. We thus pared out a lot of the detail including all of the mathematical formulae converting the unit to the appropriate combination of defined constants, and many numbers with nine or more digits. 
We can also make some other comments regarding choices we made:

Choices of units: As has often been observed in the past, the choices of units, and particularly the base units, are driven in part by two considerations: i) commonly-used units, and ii) quantities at human scales. For instance, length is an often-measured quantity, and $1 \mathrm{~m}$ is a human-scale length. These considerations drove our choices of which units to include.

Based on Technology: From the beginning of civilization, measurements and units have been based on the best available technology (e.g., using as discussed earlier a seed or a grain as the unit of mass or volume). Several of the realizations in proposal $\mathrm{C}$ depend explicitly on available technology as of 2018. This has the educational advantage of making clear to students the dependence of units on technology. It also has the disadvantage of possibly becoming dated. However, since no educational tool is ever fixed for all time, we do not view the need to update an educational adjunct as a large disadvantage; we suggest that the relative advantage is an appropriate issue for the community to discuss.

Josephson Volt and quantum Hall Ohm versus Single-Electron Ampere: One could argue that it would be conceptually simpler to base the electrical units on the clocked passage of electrons. We chose to base it on the most commonly-used technology at this time, following the logic of the previous consideration.

Explicit Dependence on Particular Experiments: Some of the realizations proposed in C (second and meter) do not have explicit reference to a particular experiment, while some of the others (volt and ohm) do. We did this to keep the definitions more widely applicable, where possible; again, this specificity is an issue that the educational community could discuss in the context of reaching consensus about an educational adjunct

The resulting proposal $\mathrm{C}$ is the one included in Figure 3, and the one that we propose as a starting point for discussion in the community.

In addition, we also note that we have included a paragraph discussing different ways to realize measurements of energy. We included this for two reasons that we believe will appeal to students: (1) It manifestly demonstrates that the relationships between various quantities in both the present and the proposed SI are largely governed by the requirement that energy derived in different ways (e.g., thermal, mechanical, mass-equivalent, quantized) must be coherent; (2) It also manifestly demonstrates the remarkable ability of the SI to allow measurements of the same quantity that vary by many many orders of magnitude. 


\section{Journal of Research of National Institute of Standards and Technology}

The International System of Units (SI) is based on assigned exact numerical values of a set of dimensional fundamental physical constants of nature. This set of constants, called the defining constants, is carefully chosen such that any unit of measure within the SI can be expressed as a subset of the defining constants. The numerical values are chosen in order to preserve continuity with earlier definitions of the metric system and SI (dating from the 1880s).

The international system of units, the SI, is the system of units in which

- $\quad$ the unperturbed ground state hyperfine splitting frequency of the caesium 133 atom $\left.\Delta v^{(133} \mathrm{Cs}\right)_{\mathrm{hfs}}$ is exactly 9 192631770 hertz,

- the speed of light in vacuum $c$ is exactly 299792458 metre per second,

- the Planck constant $h$ is exactly $6.62606957 \times 10^{-34}$ joule second,

- the elementary charge $e$ is exactly $1.602176565 \times 10^{-19}$ coulomb,

- $\quad$ the Boltzmann constant $k$ is exactly $1.3806488 \times 10^{-23}$ joule per kelvin,

- $\quad$ the Avogadro constant $N_{\mathrm{A}}$ is exactly $6.02214129 \times 10^{23}$ reciprocal mole,

- the luminous efficacy $K_{\mathrm{cd}}$ of monochromatic radiation of frequency $540 \times 10^{12}$ hertz is exactly 683 lumen per watt,

where the hertz, joule, coulomb, lumen, and watt, with unit symbols $\mathrm{Hz}, \mathrm{J}, \mathrm{C}, \mathrm{lm}$, and W, respectively, are related to the units second, metre, kilogram, ampere, kelvin, mole, and candela, with unit symbols s, m, kg, A, K, mol, and cd, respectively, according to the relations $\mathrm{Hz}=\mathrm{s}^{-1}$ (for periodic phenomena), $\mathrm{J}=\mathrm{kg} \mathrm{m}^{2} \mathrm{~s}^{-2}, \mathrm{C}=\mathrm{A} \mathrm{s}, \mathrm{lm}=\mathrm{cd} \mathrm{sr}$, and W $=$ $\mathrm{kg} \mathrm{m}^{2} \mathrm{~s}^{-3}$. The steradian, symbol sr, is the SI unit of solid angle and is a special name and symbol for the number 1 , so that $\mathrm{sr}=\mathrm{m}^{2} \mathrm{~m}^{-2}=1$.

Fig. 1. Proposal A: Defining constants. From Sec. 2.2 of the December 2013 draft of the $9^{\text {th }}$ SI Brochure [1]. 
The International System of Units (SI) is based on assigned exact numerical values of a set of dimensional fundamental physical constants of nature. This set of constants, called the defining constants, is carefully chosen such that any unit of measure within the SI can be expressed as a subset of the defining constants. The numerical values are chosen in order to preserve continuity with earlier definitions of the metric system and SI (dating from the 1880s).

The following is a list of the traditional base units expressed in terms of the defining constants.

\section{A. Second}

The second, $\mathrm{s}$, is the SI unit of time; its magnitude is set by fixing the numerical value of the ground state hyperfine splitting frequency of the caesium 133 atom, at rest and at a temperature of $0 \mathrm{~K}$, to be equal to exactly 9192631770 when it is expressed in the SI unit s ${ }^{-1}$, which for periodic phenomena is equal to $\mathrm{Hz}$.

\section{B. Metre}

The metre, $\mathrm{m}$, is the SI unit of length; its magnitude is set by fixing the numerical value of the speed of light in vacuum to be equal to exactly 299792458 when it is expressed in the SI unit $\mathrm{m} \cdot \mathrm{s}^{-1}$.

\section{Kilogram}

The kilogram, $\mathrm{kg}$, is the SI unit of mass; its magnitude is set by fixing the numerical value of the Planck constant to be equal to exactly $6.62606957 \times 10^{-34}$ when it is expressed in the SI unit $\mathrm{m}^{2} \cdot \mathrm{kg} \cdot \mathrm{s}^{-1}$, which is equal to $\mathrm{J} \cdot \mathrm{s}$.

\section{Ampere}

The ampere, A, is the SI unit of electric current; its magnitude is set by fixing the numerical value of the elementary charge to be equal to exactly $1.602176565 \times 10^{-19}$ when it is expressed in the SI unit $\mathrm{A} \cdot \mathrm{s}$, which is equal to $\mathrm{C}$.

\section{E. Kelvin}

The kelvin, symbol K, is the SI unit of thermodynamic temperature; its magnitude is set by fixing the numerical value of the Boltzmann constant to be exactly $1.3806488 \times 10^{-23}$ when it is expressed in the SI unit for energy per thermodynamic temperature $\mathrm{J} \cdot \mathrm{K}^{-1}=\mathrm{kg} \cdot \mathrm{m}^{2} \cdot \mathrm{s}^{-2} \cdot \mathrm{K}^{-1}$.

\section{F. Mole}

The mole, mol, is the SI unit of amount of substance of a specified elementary entity, which may be an atom, molecule, ion, electron, any other particle or a specified group of such particles; its magnitude is set by fixing the numerical value of the Avogadro constant to be equal to exactly $6.02214129 \times 10^{23}$ when it is expressed in the SI unit $\mathrm{mol}^{-1}$.

\section{G. Candela}

The candela, cd, is the SI unit of luminous intensity in a given direction; its magnitude is set by fixing the numerical value of the luminous efficacy of monochromatic radiation of frequency $540 \times 10^{12} \mathrm{~Hz}$ to be equal to exactly 683 when it is expressed in the SI unit $\mathrm{m}^{-2} \cdot \mathrm{kg}^{-1} \cdot \mathrm{s}^{3} \cdot \mathrm{cd} \cdot \mathrm{sr}$, or $\mathrm{cd} \cdot \mathrm{sr}^{\cdot} \mathrm{W}^{-1}$, which is equal to $\mathrm{lm} \cdot \mathrm{W}^{-1}$

Fig. 2. Proposal B: Traditional base units expressed in terms of defining constants. Adapted from Sec. 2.4 of the December 2013 draft of the $9^{\text {th }}$ SI Brochure [1]. 
The International System of Units (SI) is based on assigned exact numerical values of a set of dimensional fundamental physical constants of nature. This set of constants, called the defining constants, is carefully chosen such that any unit of measure within the SI can be expressed as a subset of the defining constants. The numerical values are chosen in order to preserve continuity with earlier definitions of the metric system and SI (dating from the 1880s).

The SI has 29 units with special names and symbols. Each unit can be realized through various experiments that link the unit to the defining constants. Below is a list of possible realizations for some of the more familiar units.

\section{A. Second}

The second is the duration of 9192631770 periods of the radiation corresponding to the transition between the two hyperfine levels of the ground state of the caesium 133 atom.

B. Metre

The metre is the length of the path travelled by light in vacuum during a time interval of 1/299 792458 of a second.

C. Volt

The volt is the electrostatic potential developed by an array of $n=10000$ Josephson junctions, which are low temperature microelectronic devices that produce a voltage when exposed to microwave radiation at frequency $f=48.35979 \mathrm{GHz}$.

D. Ohm

The ohm is $1 / 25812.807$ of the electrical resistance defined as the ratio of transverse voltage to longitudinal current in a quantum Hall resistance bar, which is a low-temperature two-dimensional microelectronics device subjected to a large magnetic field.

\section{E. Kilogram}

The kilogram is the mass $m$ that, when moved vertically in a gravitational field $g=9.8 \mathrm{~m} / \mathrm{s}^{2}$, at a velocity $v=$ $2 \mathrm{~mm} / \mathrm{s}$, generates a power of $0.0196 \mathrm{~W}$ as measured from electrical power $P=V^{2} / R$.

\section{F. Joule}

The quantity of energy relates many different units, allowing the scaling of experimental results of energy (or mass) over many orders of magnitude. Below are some energy equivalent definitions that illuminate this scalability:

1. $8.98755179 \times 10^{16}$ joule is the atomic energy produced when $1 \mathrm{~kg}$ of mass is completely converted to energy with $100 \%$ efficiency (for instance in an ideal nuclear reactor with perfect conversion and no power loss).

2. 1 joule is the energy produced by $1 \mathrm{~V}$ of voltage across a $1 \mathrm{ohm}$ resistor in one second

3. $6.212926 \times 10^{-21}$ joule is the thermal kinetic energy held in one gas monatomic atom at 300 kelvin (about room temperature).

4. $1.602176 \times 10^{-23}$ joule is the kinetic energy generated in one electron which has been accelerated in a single Josephson junction exposed to microwave radiation of frequency $f=48.35979 \mathrm{GHz}$.

Fig. 3. Proposal C: Definitions based on experiments.

\section{Questionnaire Details and Results}

We asked students to respond to an informal questionnaire at the University of Maryland, College Park. This questionnaire was given to students in a recitation section of the introductory physics sequence for physics students; students were free to attend or not attend the section, and about half of the student body (29/55) chose to attend. These students read the three proposals and gave us their numerical ratings and qualitative comments; please see Appendix Fig. 7 for the wording of the questionnaire.

The students were roughly equally split by gender, with $80 \%$ studying physics, and the rest from a variety of technical and non-technical disciplines. About half were freshmen, one third had finished one year of undergraduate studies, $15 \%$ had finished two years, and the rest more than two years.

We can quote two written comments that we found quite illuminating, in terms of relative ease of understanding between the three proposals: 
- "I preferred proposal C. This proposal was clear and expressed the SI units in terms of conceivable phenomena/measurements whereas A associated the SI units with less conceivable (however reasonable) phenomena/measurements. While proposal A was clear, it was not as helpful in connecting the quantitative aspects of the units with the qualitative aspects, i.e., the phenomena the units are measurements of."

- "I felt that the two best proposals in terms of contents were B and C. Even though they were describing similar things using constants such as the speed of light to describe a meter, I felt proposal $\mathrm{C}$ did the best job. It actually put a new value to the common SI units, which I feel is more effective than just saying what constant the SI unit relates to (seen in proposal B). If the point of this is to completely redefine SI units, proposal $\mathrm{C}$ does a much better job conveying the information and the basic theme of the set of definitions was much clearer. As for proposal A, I felt that even though the wording was a lot easier to understand, it didn't fully convey the new information and was just too simplistic."

We see from both Figures 4 and 5, and from the Table in Fig. 6, that there were significant differences in the students' rating of both (1) the clarity of the wording in the three proposals and (2) how compelling the motivation or conceptual underpinning was. Both the Figures 4 and 5 and the numerical values in the Table make it clear that the students modestly preferred proposal C over B, and both substantially over A.

As discussed earlier, we started with a much longer and more detailed proposal C; we also presented the comparison of $\mathrm{A}, \mathrm{B}$, and original $\mathrm{C}$ in an earlier questionnaire to 55 students who came from a much broader range of disciplines. The ratings in this earlier questionnaire were quite similar with respect to the motivation question; however, the students did not prefer any one proposal over the others in terms of wording. Thus, it is clear that substantially shortening and simplifying proposal $\mathrm{C}$ significantly improved students' understanding of the wording of the proposal, while not reducing their understanding of the motivation.

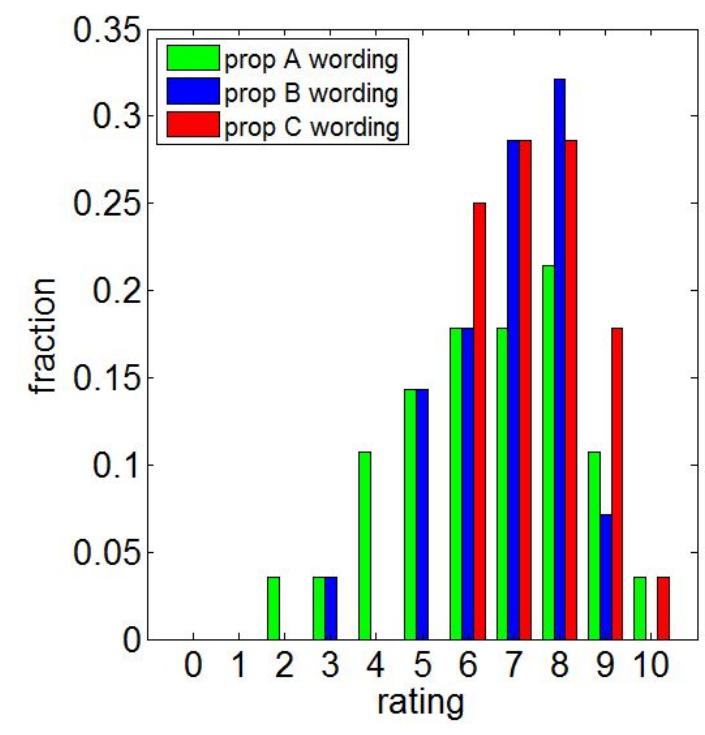

Fig. 4. Questionnaire Results: Wording. 


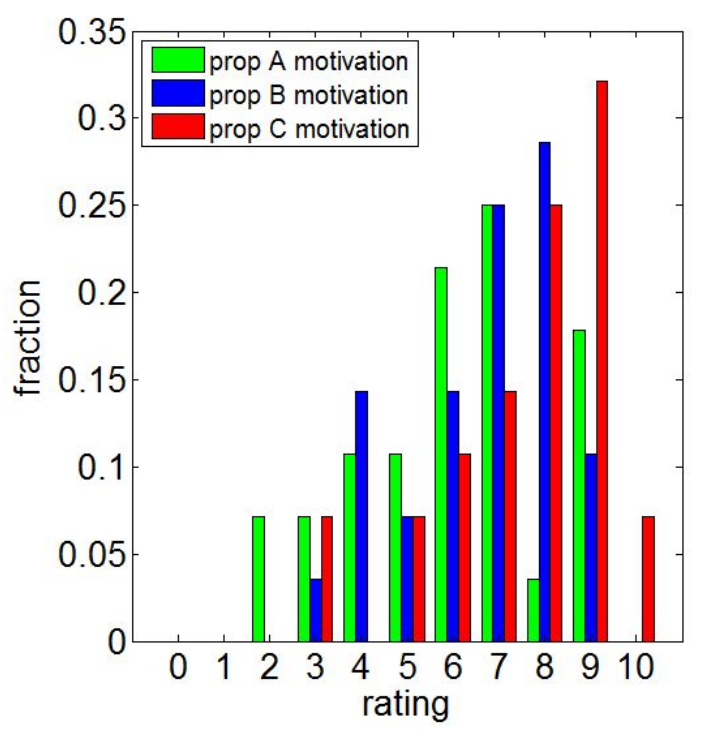

Fig. 5. Questionnaire Results: Motivation.

\begin{tabular}{|l|l|l|l|}
\hline & Proposal A & Proposal B & Proposal C \\
\hline $\begin{array}{l}\text { How well did you } \\
\text { understand the } \\
\text { wording in this } \\
\text { Proposal? }\end{array}$ & $6.5 \pm 1.9$ & $6.9 \pm 1.4$ & $7.5 \pm 1.1$ \\
\hline $\begin{array}{l}\text { How well did you } \\
\text { understand the } \\
\text { theme or motivation } \\
\text { for defining the } \\
\text { units in the way } \\
\text { used in this } \\
\text { Proposal? }\end{array}$ & $6.0 \pm 2.0$ & $6.7 \pm 1.7$ & $7.6 \pm 1.8$ \\
\hline
\end{tabular}

Fig. 6. Questionnaire Numerical Results; The uncertainties are statistical (type A) $1 \sigma$ standard deviations.

\section{Conclusions and Next Steps}

As stated in the Introduction, our original motivation for pursuing this work was our concern that, with the likely redefinition of the SI, students' initial introduction to the subject might be more challenging and less straightforward. Indeed, we believe that the informal questionnaire that we offered (informal in the sense that we did not pursue a detailed sampling algorithm, but simply let students self-select) bears out our concern: Students clearly indicated that the simple list of defined constants (proposal A) is significantly less clear in the motivation for defining a system of units in that way.

The questionnaire results also show that proposal C in Figure 3 satisfies our two main goals: (1) a clear conceptual underpinning for how to realize a system of units and (2) a simple exposition.

We do not insist that proposal $\mathrm{C}$ is the only or the best possible way to introduce students to the new SI. However, we do believe that, for high school and undergraduate teachers, it represents a useful, thought-provoking alternative to, for instance, the list of defined constants embodied in proposal A. Our 
hope is that this paper, by pointing out that the discussions in the $9^{\text {th }}$ SI brochure are not the only possible ways to introduce the new SI to students, helps to instigate a discussion amongst teachers, with the end goal of providing the best introduction to students by both teachers and textbook authors.

\section{Appendix: Questionnaire Cover Page}

\section{Questionnaire on Three Possible Textbook Redefinitions of the System of Units}

It is likely that, in 2018, the SI will be redefined so that the definitions of all units will be based on the exact values of seven fundamental physical constants. We (Neil

Zimmerman and David Newell) are considering publishing a suggestion that, in addition to the formal definitions enacted by international agreement, students, teachers, and textbook authors might consider an alternative set of definitions to be more understandable.

The crucial question for us is: For each of the three possible definitions (Proposals A, B, C), how clear is the basic theme of the set of definitions?

Name:

Level of education (finished one year of college, etc.):

Major or likely major:

Please answer the following two questions on a scale of 1 to $10 ; 1=$ didn't understand at all, 10 = completely understood.

\begin{tabular}{l|l|l|l|}
\hline & Proposal A & Proposal B & Proposal C \\
\hline $\begin{array}{l}\text { How well did you } \\
\text { understand the } \\
\text { wording in this } \\
\text { Proposal? }\end{array}$ & & & \\
\hline $\begin{array}{l}\text { How well did you } \\
\text { understand the } \\
\text { theme or motivation } \\
\text { for defining the } \\
\text { units in the way } \\
\text { used in this }\end{array}$ & & & \\
Proposal? & & & \\
\hline
\end{tabular}

We thank you very much for your assistance in this Questionnaire!

Please use the back to write anv additional comments vou might have.

Fig. 7. Cover page of questionnaire for students. 


\section{Acknowledgments}

We gratefully acknowledge all of the students who spent their time responding to the questionnaires, Chris Lobb (University of Maryland) for both his encouragement and for facilitating the second questionnaire, and Bob Abel (Olympic College) for very helpful comments.

\section{References}

[1] The December 2013 draft of the 9th SI Brochure is no longer available on the WWW; the 5 February 2018 version is available at https://www.bipm.org/utils/en/pdf/si-revised-brochure/Draft-SI-Brochure-2018.pdf. Proposal A is almost identical with the 5 February 2018 version. Proposal B is quite similar to Section 2.3.1 of the 5 February 2018 version. The newer version now has some references to other units; for example, the definition of the meter now has a reference to the second as being defined by $\Delta v_{C s}$. Also, it is likely that, soon after publication of this paper, the redefinition of the SI will be formally adopted on 20 May 2019.

[2] Newell DB (2014) A more fundamental International System of Units. Physics Today 67(7):35-41. https://doi.org/10.1063/PT.3.2448

[3] Aubrecht GJ (2012) Changes coming to the International System of Units. The Physics Teacher 50(6):338-342. https://doi.org/10.1119/1.4745684

[4] Williams JH (2014) Defining and Measuring Nature. 2053-2571 (Morgan Claypool Publishers). https://doi.org/10.1088/978-1-627-05279-5

[5] Alder K (2002) The Measure of All Things (Simon and Schuster, New York, NY, USA).

[6] Pratt JR (2014) How to weigh everything from atoms to apples using the revised SI. NCSL International Measure Journal 9:26-38.

[7] Fletcher N, Rietveld G, Olthoff J, Budovsky I, Milton M (2014) Electrical units in the new SI: Saying goodbye to the 1990 values. NCSL International Measure Journal 9:30-5.

[8] Mohr PJ, Newell DB (2010) Resource Letter FC-1: The physics of fundamental constants. American Journal of Physics 78(4):338-358. https://doi.org/10.1119/1.3279700

[9] Various mises en pratique are available at https://www.bipm.org/en/publications/mises-en-pratique/.

[10] Zimmerman NM (1998) A primer on electrical units in the Système International. American Journal of Physics 66(4):324-331. https://doi.org/10.1119/1.18859

About the authors: Neil Zimmerman works as a physicist in the Single-Atom Device Group of the Nanoscale Device Characterization Division; he has been at NIST since 1994, where he started in the Fundamental Electrical Measurements Group of the Electricity Division. He has been interested in better ways to communicate the System of Units since he started at NIST. His technical research is mostly involved with applications of single-electron and single-atom devices in standards of electrical current and quantum information.

David Newell received the Ph.D. in physics from the University of Colorado. He worked on the watt balance project at NIST in Gaithersburg, Maryland, as a National Research Council postdoctoral fellow. He became a NIST staff member in 1996 and was leader of the Fundamental Electrical Measurements Group from 2004 to 2010 and again since 2018. He has worked on measurements for the realization of micro- and nanoscale forces traceable to the SI, helped to establish the use of graphene in quantum electrical standards, and worked on the construction of a new watt balance to realize the kilogram from a fixed value of the Planck constant. He chairs the Committee on Data for Science and Technology (CODATA) Task Group on Fundamental Constants, which provided the exact values of the fundamental constants that form the foundation of the revised SI. He is a member of the Philosophical Society of Washington and a fellow of the American Physical Society.

The National Institute of Standards and Technology is an agency of the U.S. Department of Commerce. 